\title{
Influence d'injections quotidiennes de somatotropine bovine recombinée (rbST) sur la production laitière, l'ingestion et l'état nutritionnel de la chèvre en lactation
}

\author{
C Disenhaus ${ }^{1}$, J Hervieu ${ }^{1}, \mathrm{~F}$ Ternois ${ }^{1}, \mathrm{H}_{\text {Jammes }}{ }^{2}$, \\ G Kann ${ }^{2}$, D Sauvant ${ }^{1}$
}

1 INRA, station de nutrition et alimentation, 16, rue Claude-Bernard, 75231 Paris Cedex 05; ${ }^{2}$ INRA, unité d'endocrinologie moléculaire, 78352 Jouy-en-Josas Cedex, France

\begin{abstract}
Summary - Effects of daily injection of recombined bovine somatotropin (rbST) on milk production, voluntary intake and nutritional status of lactating goat. In lactating goats injected daily with rbST for 4 weeks, milk production increased significantly while energy balance decreased slightly and voluntary intake remained unchanged. Effects of injections on uraemia were significantly negative.
\end{abstract}

L'action galactopoiétique de la rbST bovine a été décrite chez la chèvre (Davis et al, 1989). Le travail présenté a pour objet de décrire les effets sur des chèvres d'injections quotidiennes de cette molécule pendant 4 semaines consécutives.

Matériel et méthodes - Dix chèvres en $10^{\ominus}$ semaine de lactation ont été placées en cases individuelles et réparties en 2 lots. Après une prépériode de 2 semaines, $5 \mathrm{mg}$ de rbST (Upjohn) reconstituée extemporanément ont été injectés quotidiennement en intramusculaire pendant 4 semaines aux animaux du lot traité (1). Les animaux du lot témoin (T) r'ont rien reçu.

Les animaux étaient nourris ad libitum avec une ration complète composée de foin de luzerne $(40 \%)$, de pulpe de betteraves surpressées $(40 \%)$, d'orge $(9,5 \%)$, d'un complément azoté $(9,5 \%)$ et d'un complément minéral et vitaminé $(1 \%)$ (valeur nutritionnelle de la ration: 0,9 UFL; $98 \mathrm{~g}$ de PDIN : $101 \mathrm{~g}$ de PDIE par $\mathrm{kg}$ de matière sèche). La consommation a été enregistrée quotidiennement et la quantité de lait brut produit $(P L)$ à chaque traite. Les taux butyreux (TB) et protéiques (TB), et les métabolites plasmatiques (glucose, urée, 3-hydroxybutyrate (BHB) et acides gras non estérifiés (AGNE)) ont été dosés une fois par semaine. Les bilans énergétique et azoté ont été calculés selon les recommandations de I'INRA (1988). Les analyses de variance-covariance ont été appliquées aux résultats moyens des 4 semaines successives de traitement $(1,2,3$ et 4$)$ en prenant en covariable la mesure de la même caractéristique pendant la prépériode.

Résultats et discussion - L'ingestion, le poids vif et l'état d'engraissement n'ont pas été modifiés par le traitement. La rbST a induit une augmentation rapide (en $24 \mathrm{~h}$ ) et soutenue de la PL. L'écart I-T s'accentue au cours du traitement (tableau I) atteignant $37 \%$ de la production de $T$ en semaine 4 . Les quantités de matières grasses produites ont significativement été augmentées 
Tableau I. Évolution des principaux paramètres.

\begin{tabular}{|c|c|c|c|c|c|c|}
\hline & \multicolumn{6}{|c|}{ Semaines } \\
\hline & & 1 & 2 & 3 & 4 & Moyenne \\
\hline Matièle sèche & $\mathrm{m}$ & 2370 & 2420 & 2420 & 2320 & 2380 \\
\hline $\begin{array}{l}\text { ingérée }(g / j) \\
\text { Lait }(g / j)\end{array}$ & $\begin{array}{l}d \\
m\end{array}$ & $\begin{array}{r}-30 \\
2790^{b}\end{array}$ & $\begin{array}{l}-30 \\
2750^{d}\end{array}$ & $\begin{array}{l}-10 \\
2700^{d}\end{array}$ & $\begin{array}{r}+30 \\
2620^{d}\end{array}$ & $2715^{d}$ \\
\hline & d & 434 & 618 & 848 & 972 & 718 \\
\hline $\begin{array}{l}\text { Matières grasses } \\
(g / j)\end{array}$ & $\underset{\mathrm{d}}{\mathrm{m}}$ & $\begin{array}{l}66,5^{\mathrm{b}} \\
24\end{array}$ & $\begin{array}{l}69,5^{\mathrm{d}} \\
19\end{array}$ & $\begin{array}{l}65,5^{c} \\
30\end{array}$ & $\begin{array}{l}66,0^{c} \\
38\end{array}$ & $\begin{array}{l}66,8^{\mathrm{c}} \\
27,8\end{array}$ \\
\hline $\begin{array}{l}\text { Matières protéiques } \\
(\mathrm{g} / \mathrm{i})\end{array}$ & $\mathrm{m}$ & $76,0^{b}$ & $73,0^{\circ}$ & $73,0^{6}$ & $69,5^{b}$ & $73,0^{\mathrm{b}}$ \\
\hline $\begin{array}{l}(\mathrm{g} / \mathrm{J}) \\
\text { Bilan énergétique }\end{array}$ & $\begin{array}{l}\mathrm{d} \\
\mathrm{m}\end{array}$ & $\begin{array}{l}8,0 \\
0,38\end{array}$ & $\begin{array}{c}14,0 \\
0,41\end{array}$ & $\begin{array}{r}22,0 \\
0,45\end{array}$ & $\begin{array}{r}25,0 \\
0,38\end{array}$ & \\
\hline (UFL/j) & d & $-0,14$ & $-0,15$ & $-0,23$ & $-0,27$ & \\
\hline $\begin{array}{l}\text { Bilan azoté } \\
\text { (g PDl/j) }\end{array}$ & d & $\begin{array}{r}76 \\
-14\end{array}$ & $\begin{array}{r}81 \\
-20\end{array}$ & $\begin{array}{r}82 \\
-34\end{array}$ & -35 & \\
\hline $\begin{array}{l}\text { AGNE } \\
\text { (meg/l) }\end{array}$ & $\mathrm{m}$ & 78 & $87^{a}$ & $132^{a}$ & $93^{a}$ & \\
\hline $\begin{array}{l}(\mathrm{meq} / \mathrm{l}) \\
\mathrm{BHB}(\mathrm{mg} / \mathrm{l})\end{array}$ & d & 51 & 89 & 95 & 185 & \\
\hline BHB (mg/l) & $\begin{array}{l}\mathbf{m} \\
\mathbf{d}\end{array}$ & $\begin{array}{r}37,8 \\
3,5\end{array}$ & $\begin{array}{r}33,6 \\
4,2\end{array}$ & $\begin{array}{l}37 \\
0,2\end{array}$ & $\begin{array}{r}33,2 \\
9,6\end{array}$ & \\
\hline Glucose $(\mathrm{mg} / \mathrm{l})$ & $\mathrm{m}$ & 543 & 482 & 477 & 502 & \\
\hline & d & 17 & -15 & 12 & -22 & \\
\hline Urée $(\mathrm{mg} /$ ) & $\mathrm{m}$ & $430^{d}$ & $437^{\circ}$ & $456^{\mathrm{b}}$ & $452^{a}$ & \\
\hline & d & -96 & -79 & -125 & -86 & \\
\hline
\end{tabular}

$\mathrm{m}=$ Moyenne du lot $\mathrm{T} ; \mathrm{d}=$ différence ajustée entre $\mathrm{I}$ et $\mathrm{T}$; seuils de signification : ${ }^{\mathrm{a}} P<0,1 ;{ }^{\mathrm{b}} P<0,5 ;{ }^{\mathrm{c}} P<0,01 ;{ }^{\mathrm{d}} P<0,005$.

en même temps que la $\mathrm{PL}$ (semaine 1, $2,3,4)$. Seuls le TB en $S 4(+4 \mathrm{~g} / \mathrm{l}$ : $P<0,05)$ et le TP en S1 $(-1,65 \mathrm{~g} / \mathrm{l})$ ont été significativement modifiés.

L'écart I-T des bilans énergétique et azot:: a toujours très fortement évolué en défaveur du lot 1 .

La glycémie, les teneurs en BHB et l'hématocrite ne sont pas modifiés par le traitement. L'écart I-T de la teneur en AGNE tend à augmenter au cours du traitement $(P<0,1)$. L'urémie a été nettement diminuée. Ce résultat confirme la tendance constatée sur chèvres lors d'un traitement au 1-29GRF (Sauvant et al, 1988) où l'accroissement moyen de $\mathrm{PL}$ obtenu était moindre $(8,4 \%$ vs $26,4 \%$ ) que dans le présent essai.
En conclusion, les injections quotidiennes de rbST ont significativement et durablement favorisé les productions mammaires. Les modifications des principales caractéristiques zootechniques et plasmatiques semblent similaires à celles observées chez la vache (Chilliard, 1988). La chèvre apparaît comme un modèle intéressant d'exploration des mécanismes d'action de la rbST sur la galactopoï̀se.

Chilliard Y (1988) Reprod Nutr Dev 28 (1), 39-59

Sauvant D, Kann G, Hervieu J, Disenhaus C (1988) Reprod Nutr Dev 28 (suppl 1), 179-180

Davis SR, Gluckman PD, Hodgkinson C, Farr VC, Breier BH, Burleigh BD (1989) J Endocrinol 123, 33-39 